\title{
MINERALOGIA DE UM LATOSSOLO VERMELHO DISTRÓFICO SUBMETIDO A DIFERENTES MANEJOS POR $24 \operatorname{ANOS}^{(1)}$
}

\author{
Daniele Gonçalves ${ }^{(2)}$, Wellington Claiton Leite ${ }^{(3)}$, André Maurício \\ Brinatti $^{(4)}$, Sérgio da Costa Saab ${ }^{(4)}$, Kelly Cristiane Iarosz ${ }^{(2)}$, Yvone \\ Primerano Mascarenhas ${ }^{(5)}$, Paulo Irajara Borba Carneiro' ${ }^{(6)} \&$ \\ Jadir Aparecido Rosa ${ }^{(7)}$
}

\begin{abstract}
RESUMO
Atualmente, procuram-se sistemas de manejo que preservem ou melhorem as qualidades do solo, tanto mineralógicas como orgânicas, priorizando maior produtividade. Este trabalho teve como objetivo verificar qualitativamente, por meio das técnicas de fluorescência de raios $\mathrm{X}$, difração de raios $\mathrm{X}$ e infravermelho com transformada de Fourier, os efeitos provocados pela utilização dos manejos: plantio convencional, preparo mínimo e plantio direto, por 24 anos, na mineralogia de um Latossolo Vermelho distrófico de Ponta Grossa, PR. Não foram observadas mudanças significativas na mineralogia do Latossolo estudado, por meio das técnicas utilizadas, quanto aos diferentes manejos durante os anos de sua aplicação. Pôde-se verificar, qualitativamente, a presença dos minerais gibbsita, caulinita, haloisita, montmorilonita, hematita, rutilo, anatásio, goethita e quartzo.
\end{abstract}

Termos de indexação: plantio convencional, preparo mínimo, plantio direto, mineralogia.

\footnotetext{
(1) Trabalho apresentado no XXXI Congresso Brasileiro de Ciência do Solo, 2007 (Gramado, RS).

(2) Mestranda, Química Aplicada - Universidade Estadual de Ponta Grossa (UEPG). Av. Gal. Carlos Cavalcanti, 4748, CEP 84030900, Ponta Grossa, PR. E-mails: dagonc@gmail.com; kiarosz@gmail.com

(3) Aluno, Bacharelado em Física, Departamento de Física - UEPG, Bolsista PIBIC Av. Gal. Carlos Cavalcanti, 4748, CEP 84030900, Ponta Grossa, PR. Email: wellingtonrch@hotmail.com

(4) Professor, Departamento de Física - UEPG. E-mails: brinatti@uepg.br; scsaab@fisica.uepg.br

(5) Professor, Instituto de Física de São Carlos - Universidade de São Paulo - IFSC-USP, Caixa Postal 369, CEP13560-970, São Carlos, SP. E-mail: yvonne@ifsc.usp.br

(6) Professor, Departamento de Química - UEPG. E-mail: pibc@uepg.br

(7) Pesquisador - Instituto Agrnômico do Paraná-IAPAR - Pólo Regional de Ponta Grossa, BR 376, Km 496, CEP 84001-970, Ponta Grossa, PR. E-mail: jrosa@iapar.br
} 


\title{
SUMMARY: MINERALOGY OF A RED LATOSOL UNDER DIFFERENT MANAGEMENT SYSTEMS FOR TWENTY-FOUR YEARS.
}

\begin{abstract}
The search for management systems that preserve or improve soil qualities, the mineralogical as well as the organic composition, with a view to higher yields, is becoming more and more intense. The objective this study was to qualitatively verify the mineralogical effects of different management types: conventional, minimum and no-tillage for 24 years on a Red Latosol in Ponta Grossa, PR, Brazil, by the following techniques: X-ray diffraction, $X$-ray fluorescence and Fourier transform infrared. No significant changes in the mineralogy of the Red Latosol were observed by the techniques applied, as a result of the different management types. It was however possible to qualitatively verify the presence of the following minerals: gibbsite, kaolinite, halloysite, montmorillonite, hematite, rutile, anatase, goethite and quartz.
\end{abstract}

Index terms: conventional tillage, minimum tillage, no-tillage, mineralogy.

\section{INTRODUÇÃO}

Nos últimos anos, tem sido mais freqüente a procura por sistemas de manejo que conservem o solo ou que resultem em sua melhoria. Isto se deve à busca de maiores produtividades e ao aumento da população mundial e, em decorrência, à maior demanda por alimentos (Silva \& Silveira, 2002; Bronick \& Lal, 2005).

Assim, a importância de escolher sistemas de manejo mais adequados ao solo está na contribuição que podem trazer para a melhoria de suas qualidades, mantendo ou alterando ao mínimo suas características e propriedades (Ribon et al., 2002; Costa et al., 2003), as quais estão relacionadas com sua composição mineralógica (Reatto et al., 1998; Ribon et al., 2002; Costa et al., 2003) e orgânica (Bayer et al., 2003).

O Paraná, por ser um Estado eminentemente agrícola (MacCulloch, 1999), merece uma avaliação do efeito dos diferentes sistemas de manejos sobre a composição mineralógica do solo. A região de Ponta Grossa, PR, nos Campos Gerais, foi pioneira na adoção do plantio direto (PD) como forma de manejo (IAPAR, 2007) e sua utilização vem crescendo consideravel-mente, em muitas áreas, mesmo naquelas utilizadas anteriormente como pastagens naturais para exploração pecuária (Rheinheimer et al., 2000).

Os sistemas de manejo PD e preparo mínimo (PM) são conservacionistas, ou métodos não-convencionais, pois mantêm no solo restos de culturas anteriores e evitam sua erosão. Ainda, aumentam a disponibilidade de nutrientes e de matéria orgânica, melhorando as condições do solo e, conseqüentemente, contribuindo para a produção agrícola (Silva et al., 2001; Schaefer et al., 2001; Cavalieri et al., 2004). Em contrapartida, o manejo plantio convencional (PC) favorece a erosão graças ao intenso revolvimento do solo e a falta de cobertura aumenta sua degradação (Rheinheimer et al., 1998).

O tempo de utilização de um sistema de manejo deve ser considerado para avaliar os efeitos ocasionados à qualidade do solo (Costa et al., 2003), relativos não só à avaliação das respostas cumulativas, como a estabilidade da matéria orgânica e as interações com componentes minerais (Bayer, 1996), mas também à influência da agregação e estabilidade estrutural do solo nos atributos físicos e químicos (Silva \& Mielniczuk, 1998) não observados nos anos iniciais, efeitos esses, do experimento (Pauletti et al., 2003) ou da aplicação do sistema manejo.

Este trabalho teve como objetivo avaliar, por meio das técnicas de fluorescência de raios X (FRX), difração de raios X (DRX) e infravermelho com transformada de Fourier (IV), os efeitos provocados qualitativamente na mineralogia do solo quanto a modificações das quantidades dos óxidos potencialmente reativos, como óxidos de $\mathrm{Fe}$ e caulinita (Baldock et al., 1992), pela utilização dos manejos PC, PD e PM aplicados por 24 anos em um Latossolo Vermelho distrófico de Ponta Grossa, PR.

A FRX foi utilizada por ser um dos métodos instrumentais rotineiros empregados nas análises de elementos em rochas, cimentos, dentre outros (Mori et al., 1999), e permitir determinar o teor de elementos numa amostra, utilizando as intensidades de raios $\mathrm{X}$ característicos emitidos, possibilitando a detecção simultânea de elementos numa ampla faixa de número atômico e teores.

Já a DRX é uma técnica de grande versatilidade e rapidez na aplicação de amostras policristalinas, tais como o monitoramento de amostras em laboratório, no controle de qualidade industrial e identificação 
mineralógica em solos e rochas (Weidler et al., 1998; Esteve et al., 2000; Albers et al., 2002; Ferreira et al., 2003).

Por fim, o IV é complementar à DRX no reconhecimento mineral (Gadsden, 1975), por fornecer informações relevantes quanto à identificação, quantificação e aos aspectos estruturais dos minerais argilosos, cristalinos ou amorfos (Gomes, 1986) em amostras de solo, além de ser uma técnica rápida, simples e econômica (Farmer, 1974; Gadsden, 1975).

\section{MATERIAL E MÉTODOS}

O solo analisado é um Latossolo Vermelho distrófico proveniente da Estação Experimental do Instituto Agronômico do Paraná (IAPAR), latitude $25^{\circ} 13^{\prime}$ S e longitude $50^{\circ} 01^{\prime} \mathrm{W}$; precipitação pluvial total anual entre 1.300 a $1.800 \mathrm{~mm}$, com chuvas bem distribuídas ao longo do ano; estações climáticas bem definidas termicamente como média do mês mais quente (fevereiro) $21,2^{\circ} \mathrm{C}$ e do mais frio (julho) $13,3{ }^{\circ} \mathrm{C}$ (Merten, 1995; Medeiros \& Melo, 2001). Nas parcelas experimentais, duas com dimensão de 100 x $50 \mathrm{~m}$ e a terceira de $100 \mathrm{x}$ $100 \mathrm{~m}$, com declividades de 6,$9 ; 7,1 ; 8,1 \%$, respectivamente, foram aplicados os seguintes sistemas de manejos: PC, PM e PD, com rotações de cultura desde o início do experimento, em 1981, até o ano de 2005 (Merten, 1995).

Amostras de solo foram retiradas de quatro pontos diferentes das parcelas dos manejos e foram individualmente homogeneizadas. Em seguida, realizou-se a secagem a $70{ }^{\circ} \mathrm{C}$ por $24 \mathrm{~h}$ e, posteriormente, as amostras foram peneiradas em malha de $0,001 \mathrm{~m}$. As amostras foram coletadas nas profundidades de 0,0-0,20 m, 0,20-0,40 m e 0,40-0,60 $\mathrm{m}$; nos manejos: PC, PM e PD; totalizando nove amostras: am01 (PC 0,0-0,20 m), am02 (PC 0,20-0,40 $\mathrm{m}), \operatorname{am03}(\mathrm{PC}$ 0,40-0,60 m), am04 (PM 0,0-0,20 m), am05 (PM 0,20-0,40 m), am06 (PM 0,40-0,60 m), am07 (PD 0,0-0,20 m), am08 (PD 0,20-0,40 m) e am09 (PD 0,40-0,60 m).

As amostras foram maceradas em almofariz e tamisadas em peneiras de abertura de $53 \mu \mathrm{m}$ para garantir a homogeneidade dos tamanhos de partículas nas análises de FRX, IV e DRX. Essas etapas iniciais de preparação das amostras (peneiramento e pesagem) foram realizadas no Laboratório de Física Aplicada a Solos e Ciências Ambientais (FASCA), Departamento de Física da UEPG.

As nove amostras oriundas das três profundidades foram analisadas por FRX. Foram preparadas pastilhas fundidas de acordo com o protocolo de Mori et al. (1999), utilizando $2 \mathrm{~g}$ de amostra em cada uma, sendo as análises realizadas no Laboratório de Fluorescência de Raios X do DMPIG da USP.
No IV, as pastilhas foram confeccionadas na proporção de $1 \mathrm{mg}$ de cada amostra para $100 \mathrm{mg}$ de $\mathrm{KBr}$, devidamente macerados para garantir uma mistura bem homogênea e utilizando um pastilhador e prensa (pressão de 60 Gauge - aproximadamente $6 \mathrm{t}$ por $30 \mathrm{~s})$. As medidas de IV feitas com um espectrofotômetro Shimadzu, modelo FTIR-8400, operando no modo Fourier Transform, na resolução de $4 \mathrm{~cm}^{-1}$, na faixa do infravermelho médio (4000 a $400 \mathrm{~cm}^{-1}$ ), em 16 varreduras, no Laboratório de Química Analítica e Orgânica, no Departamento de Química da UEPG. A identificação dos minerais por meio de suas bandas de vibração características foi realizada com o auxílio de tabelas (Farmer, 1974; Gadsden, 1975).

Nas análises de DRX, cada amostra foi colocada em um porta-amostra e pressionada suavemente para evitar possíveis orientações preferenciais. A coleta de dados ocorreu com a utilização de um difratômetro Rigaku Rotaflex RU200B (12kW); tubo com anodo rotatório; goniômetro RINT 2000 Wide Angle Goniometer, no modo varredura contínua. As condições de operação foram: extensão $(\theta-2 \theta): 3^{\circ} \mathrm{a}$ $100^{\circ} ; 2,0$ \% min; radiação CuKo; $50 \mathrm{kV} ; 100 \mathrm{m \AA}$; fendas de: divergência $=1,0^{\circ}$; espalhamento $=1,0^{\circ}$; recepção $=0,30 \mathrm{~mm}$. Essa etapa foi realizada no Laboratório de Cristalografia do Instituto de Física de São Carlos da USP.

\section{RESULTADOS E DISCUSSÃO}

Os dados obtidos pela FRX, teores de óxidos, são apresentados no quadro 1. Esses indicam a presença predominante dos óxidos de Al, Si, Fe e Ti em ordem decrescente, e, ainda, em proporções menores, os óxidos de $\mathrm{Mg}, \mathrm{Mn}, \mathrm{P}, \mathrm{Ca}, \mathrm{K}$ e $\mathrm{Na}$ nas amostras analisadas. A partir desses dados, calcularam-se os teores de cada elemento nas amostras (Quadro 1), e essas informações auxiliaram na identificação dos minerais por meio dos óxidos com maiores teores por ela indicados e na análise de modificações mineralógicas pouco significativas quanto ao tipo de manejo.

Os espectros de IV obtidos para as amostras não apresentaram mudanças quanto ao tipo de manejo (Figura 1). Variações na absorbância são aceitas, pelo fato de tratar-se de uma análise qualitativa. Os espectros de IV permitiram identificar as bandas, que indicam presença dos seguintes minerais: gibbsita, grupo caulim (caulinita e haloisita), montmorilonita e quartzo, de acordo com a literatura (Farmer, 1974; Gadsden, 1975), $3530 \mathrm{~cm}^{-1}, 3446 \mathrm{~cm}^{-1}$ e $3387 \mathrm{~cm}^{-1}$ para gibbsita; $3696 \mathrm{~cm}^{-1}$ e $3621 \mathrm{~cm}^{-1}$ para o grupo caulim; $1037 \mathrm{~cm}^{-1}, 921 \mathrm{~cm}^{-1}$ e $474 \mathrm{~cm}^{-1}$ para a montmorilonita; $800 \mathrm{~cm}^{-1}$ e $731 \mathrm{~cm}^{-1}$ para o quartzo. 
Quadro 1. Teores dos óxidos e respectivos elementos (referentes ao cátion constituinte do óxido) nas amostras de solo, nos diferentes manejos e profundidades obtidos por FRX

\begin{tabular}{|c|c|c|c|c|c|c|c|c|c|c|c|}
\hline Amostra & Óxidos & $\mathrm{Al}_{2} \mathrm{O}_{3}$ & $\mathrm{SiO}_{2}$ & $\mathrm{Fe}_{2} \mathrm{O}_{3}$ & $\mathrm{TiO}_{2}$ & MgO & MnO & $\mathbf{P}_{2} \mathbf{O}_{5}$ & $\mathrm{CaO}$ & $\mathbf{K}_{2} \mathbf{O}$ & $\mathrm{Na}_{2} \mathrm{O}$ \\
\hline \multicolumn{12}{|c|}{$\mathrm{g} \mathrm{kg}^{-1}$} \\
\hline \multicolumn{12}{|c|}{ Profundidade de $0,0-0,20 \mathrm{~m}$} \\
\hline \multirow{2}{*}{$\mathrm{am} 01$} & Óxidos & 349,90 & 257,70 & 129,90 & 20,78 & 3,20 & 0,28 & 2,30 & 1,60 & 5,00 & 0,70 \\
\hline & Elemento $^{(1)}$ & 92,59 & 120,46 & 45,43 & 12,50 & 1,93 & 0,22 & 0,50 & 1,14 & 2,08 & 0,26 \\
\hline \multirow{2}{*}{ am04 } & Óxidos & 350,30 & 248,80 & 127,90 & 21,04 & 3,30 & 0,35 & 2,83 & 2,40 & 3,80 & 0,50 \\
\hline & Elemento $^{(1)}$ & 92,70 & 116,30 & 44,73 & 12,60 & 1,99 & 0,27 & 0,62 & 1,72 & 1,58 & 0,19 \\
\hline \multirow{2}{*}{$\operatorname{am} 07$} & Óxidos & 343,40 & 241,70 & 128,40 & 21,04 & 3,30 & 0,31 & 2,73 & 1,90 & 4,20 & 0,90 \\
\hline & Elemento $^{(1)}$ & 90,87 & 112,98 & 44,90 & 12,60 & 1,99 & 0,24 & 0,60 & 1,36 & 1,74 & 0,33 \\
\hline \multicolumn{12}{|c|}{ Profundidade de $0,20-0,40 \mathrm{~m}$} \\
\hline \multirow{2}{*}{ am02 } & Óxidos & 346,30 & 263,70 & 129,30 & 20,62 & 3,60 & 0,27 & 2,16 & 1,60 & 5,30 & 0,40 \\
\hline & Elemento $^{(1)}$ & 91,64 & 123,26 & 45,22 & 12,40 & 2,17 & 0,20 & 0,47 & 1,14 & 2,20 & 0,15 \\
\hline \multirow{2}{*}{$\operatorname{am} 05$} & Óxidos & 356,80 & 248,90 & 130,20 & 21,37 & 3,10 & 0,33 & 2,07 & 1,60 & 3,40 & 0,50 \\
\hline & Elemento $^{(1)}$ & 94,42 & 116,35 & 45,53 & 12,80 & 1,87 & 0,26 & 0,45 & 1,14 & 1,41 & 0,19 \\
\hline \multirow{2}{*}{$\operatorname{am08}$} & Óxidos & 353,00 & 251,80 & 127,70 & 21,50 & 3,10 & 0,27 & 1,97 & 1,30 & 4,60 & 1,10 \\
\hline & Elemento $^{(1)}$ & 93,41 & 111,70 & 44,66 & 12,90 & 1,87 & 0,20 & 0,43 & 0,93 & 1,91 & 0,41 \\
\hline \multicolumn{12}{|c|}{ Profundidade de $0,40-0,60 \mathrm{~m}$} \\
\hline \multirow{2}{*}{ am03 } & Óxidos & 355,10 & 258,80 & 132,70 & 20,76 & 3,30 & 0,27 & 1,26 & 0,90 & 5,40 & 0,60 \\
\hline & Elemento $^{(1)}$ & 93,97 & 120,97 & 46,41 & 12,40 & 1,99 & 0,20 & 0,27 & 0,64 & 2,24 & 0,22 \\
\hline \multirow{2}{*}{$\operatorname{am} 06$} & Óxidos & 364,20 & 246,50 & 134,50 & 22,02 & 3,00 & 0,34 & 1,72 & 1,50 & 2,90 & 0,50 \\
\hline & Elemento $^{(1)}$ & 96,38 & 115,22 & 47,04 & 13,20 & 1,80 & 0,26 & 0,38 & 1,07 & 1,20 & 0,19 \\
\hline \multirow{2}{*}{ am09 } & Óxidos & 365,50 & 247,30 & 132,40 & 22,38 & 2,80 & 0,31 & 1,32 & 0,80 & 3,20 & 0,4 \\
\hline & Elemento ${ }^{(1)}$ & 96,72 & 115,60 & 46,30 & 13,40 & 1,69 & 0,24 & 0,29 & 0,57 & 1,33 & 0,15 \\
\hline
\end{tabular}

(1) Refere-se ao cátion constituinte do respectivo óxido.
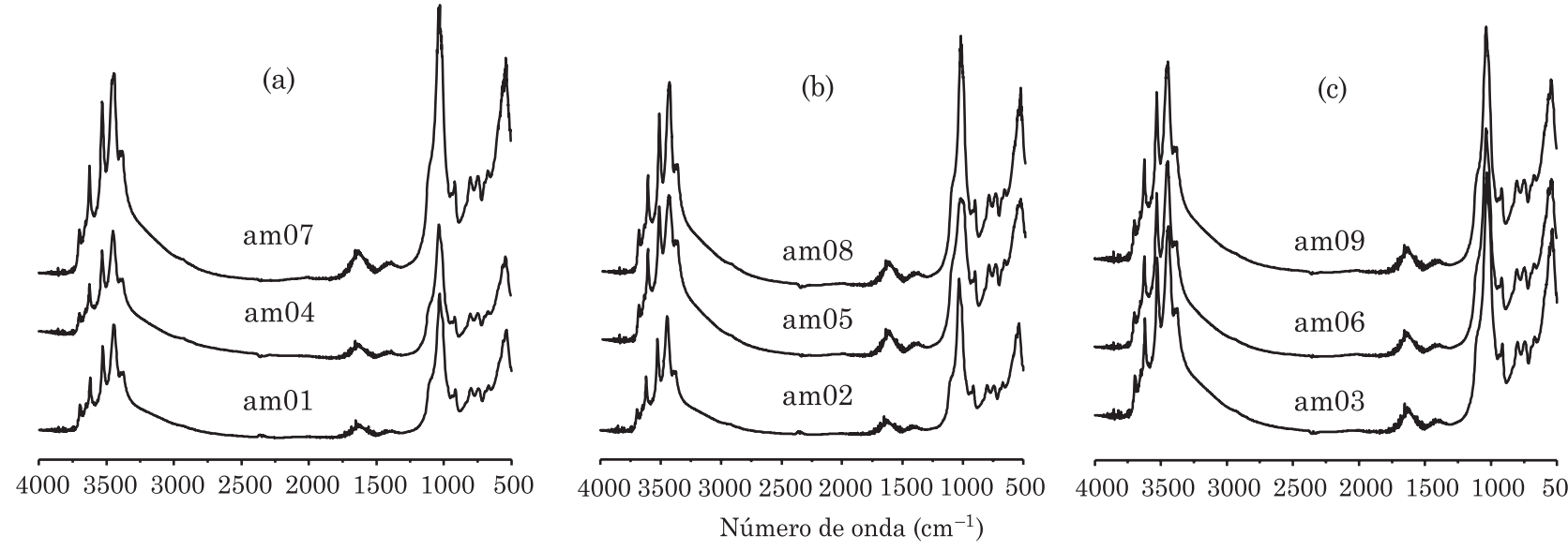

4000350030002500200015001000500

Número de onda $\left(\mathrm{cm}^{-1}\right)$

Figura 1. Espectros obtidos por IV para as nove amostras analisadas nos diferentes manejos e profundidades. Profundidades: (a) 0,0-0,20 m, (b) 0,20-0,40 m e (c) 0,40-0,60 m; em cada um dos gráficos, os espectros do inferior ao superior representam os manejos: PC, PM e PD, respectivamente. 
Os difratogramas não apresentaram mudanças significativas quanto ao tipo de manejo e profundidade; apenas as intensidades, quando verificadas, aparecem com alguma diferenciação, tanto de um manejo para outro como entre as profundidades (Figura 2).
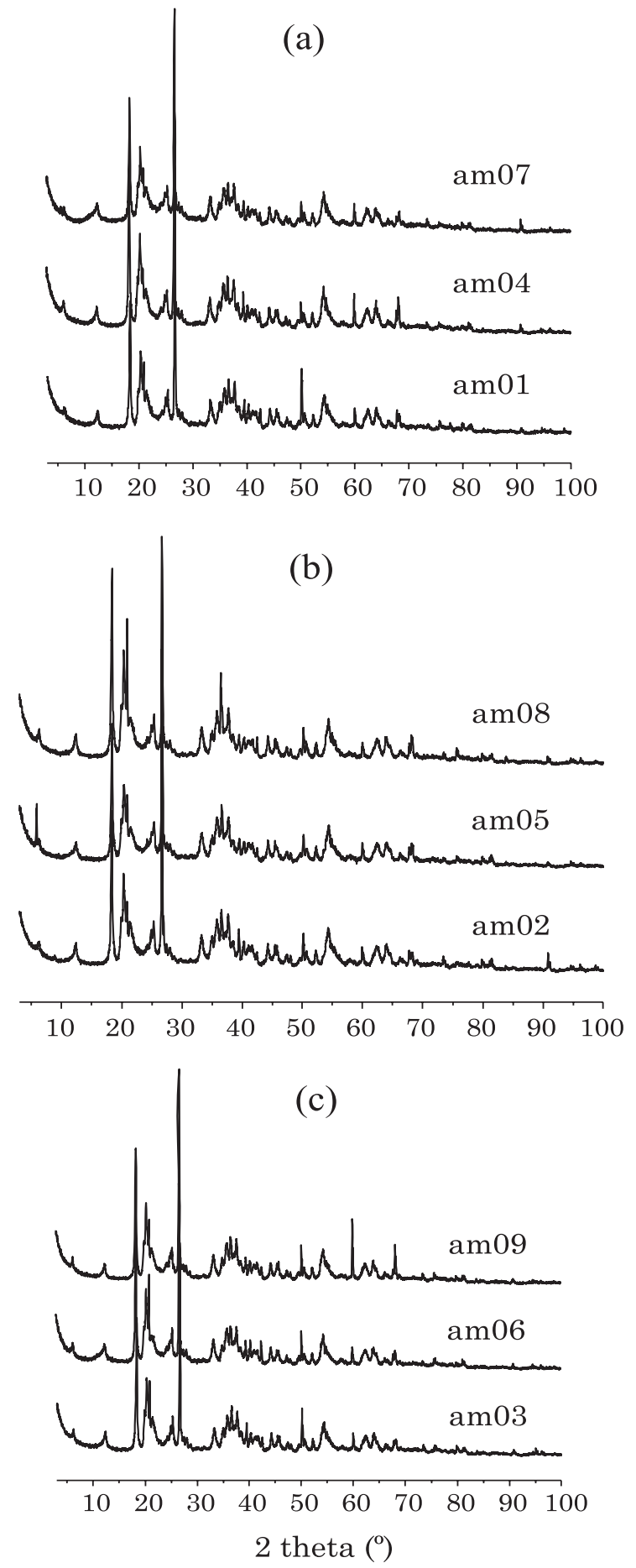

Figura 2. Difratogramas das nove amostras analisadas nos diferentes manejos e profundidades. Profundidades: (a) 0,0-0,20 m, (b) 0,20-0,40 $\mathrm{m}$ e (c) 0,40 - 0,60 m; em cada um dos gráficos, os difratogramas do inferior ao superior representam os manejos: PC, PM e PD, respectivamente.
Entretanto, os picos de difração mais intensos para cada amostra, de acordo com a literatura (Moore \& Reynolds, 1997; Brindley \& Brown, 1980), indicaram a presença dos minerais: gibbsita $(d=4,84 \AA, 2 \theta=$ $\left.18,33^{\circ}\right)$, caulinita $\left(\mathrm{d}=7,17 \AA, 2 \theta=12,34^{\circ} ; \mathrm{d}=3,58 \AA\right.$, $\left.2 \theta=24,94^{\circ} ; \mathrm{d}=4,37 \AA, 2 \theta=20,31^{\circ}\right)$, haloisita $(\mathrm{d}=$ $4,46 \AA, 2 \theta=19,92^{\circ} ; d=7,17 \AA, 2 \theta=12,34^{\circ} ; d=3,68 \AA$, $\left.2 \theta=24,20^{\circ}\right)$, montmorilonita $\left(\mathrm{d}=15,03 \AA \mathrm{\AA}, 2 \theta=5,88^{\circ}\right)$, hematita $\left(\mathrm{d}=2,69 \AA, 2 \theta=33,25^{\circ} ; \mathrm{d}=2,51 \AA, 2 \theta=35,83^{\circ}\right.$; $\left.\mathrm{d}=1,69 \AA, 2 \theta=54,33^{\circ}\right)$, rutilo $\left(\mathrm{d}=3,25 \AA, 2 \theta=27,47^{\circ} ; \mathrm{d}=\right.$ $\left.1,69 \AA, 2 \theta=54,37^{\circ}\right)$, anatásio $\left(\mathrm{d}=3,52 \AA, 2 \theta=25,32^{\circ}\right.$; $\left.\mathrm{d}=1,89 \AA, 2 \theta=48,02^{\circ} ; \mathrm{d}=2,39 \AA 2,2 \theta=37,70^{\circ}\right)$, goethita ( $d=2,46 \AA, 2 \theta=36,59^{\circ} ; \mathrm{d}=2,69 \AA 2,2 \theta=33,25^{\circ}$ ) e quartzo $\left(d=3,61 \AA, 2 \theta=26,66^{\circ} ; d=4,25 \AA, 2 \theta=\right.$ $\left.20,88^{\circ}\right)$.

Relacionando os resultados obtidos por meio do IV, das respectivas bandas de vibração dos minerais com os resultados obtidos pela FRX, dos elementos constituintes do solo a partir dos óxidos de Al, $\mathrm{Si}, \mathrm{Fe}$ e $\mathrm{Ti}$, tem-se um reforço da presença de gibbsita, grupo caulim (caulinita e haloisita), montmorilonita e quartzo. Acrescentando os resultados da DRX, pôde-se verificar a presença de anatásio, rutilo, hematita e goethita, além dos já citados anteriormente. Porém, não há evidências de mudanças significativas, nos três manejos estudados, observadas de forma qualitativa, na mineralogia do solo quanto aos óxidos potencialmente reativos que influenciam a estabilidade da matéria orgânica, a agregação e a estabilidade estrutural do solo.

\section{CONCLUSÕES}

1. Não foram observadas mudanças significativas na mineralogia do Latossolo Vermelho distrófico decorrentes dos diferentes sistemas de manejos durante 24 anos.

2. Em verificação qualitativa, os minerais presentes neste solo foram gibbsita, caulinita, haloisita, montmorilonita, hematita, rutilo, anatásio, goethita e quartzo.

\section{AGRADECIMENTOS}

À CAPES, pelo suporte financeiro por meio do PROAP e ao PIBIC- CNPQ-UEPG, pela bolsa de iniciação científica.

\section{LITERATURA CITADA}

ALBERS, A.P.F.; MELCHIADES, F.G.; MACHADO, R.; BALDO, J.B. \& BOSCHI, A.O. Um método simples de caracterização de argilominerais por difração de raios X. Cerâmica, 48:34-37, 2002 
BALDOCK, J.A.; OADES, J.M.; WATERS, A.G.; PENG, X.; VASSALO, A.M. \& WILSON, M.A. Aspects of the chemical structrure of soil organic material as revealed by solid-state 13C NMR spectroscopy. Biogeochemistry, 16:1-42, 1992.

BAYER, C. Dinâmica da matéria orgânica em sistemas conservacionistas de manejo de Solos. Porto Alegre, Universidade Federal do Rio Grande do Sul, 1996. 241p. (Tese de Doutorado)

BAYER, C.; MARTIN-NETO, L. \& SAAB, S.C. Diminuição da humificação da matéria orgânica de um Cambissolo Húmico em plantio direto. R. Bras. Ci. Solo, 27:537-544, 2003.

BRINDLEY, G.W. \& BROWN, G., eds. Crystal structures of clay minerals and their X-ray identification. London, Mineralogical Society, 1980. 495p.

BRONICK, C.J. \& LAL, R. Soil structure and management: A review. Geoderma, 124:3-22, 2005.

CAVALIERI, K.M.V.; TORMENA, C.A.; FIDALSKI, J.; PINTRO, J.C.; COSTA, A.C.S. \& SOUZA JR, I.G. Alterações nas propriedades químicas de um Latossolo Vermelho eutroférrico por dois sistemas de manejo de solo. Acta Sci. Agron., 26:377-385, 2004.

COSTA, F.S.; ALBUQUERQUE, J.A.; BAYER, C.; FONTOURA, S.M.V. \& WOBETO, C. Propriedades físicas de um Latossolo Bruno afetadas pelos sistemas plantio direto e preparo convencional. R. Bras. Ci. Solo, 27:527-535, 2003.

ESTEVE, V.; OCHANDO, L.E.; REVENTÓS, M.M.; PERIS, G. \& AMIGÓ, J.M. Quantitative phase analysis of mixtures of three components using rietveld and Rius Standardless Methods. Comparative results. Cryst. Res. Technol., 35:1183-1192, 2000.

FARMER, V.C., ed. The infrared spectra of minerals. London, Mineralogical Society, 1974. 539p.

FERREIRA, B.A.; FABRIS, J.D.; SANTANA, D.P. \& CURI, N. Óxidos de ferro das frações areia e silte de um Nitossolo desenvolvido de basalto. R. Bras. Ci. Solo, 27:405-413, 2003.

GADSDEN, J.A. Infrared spectra of minerals and related inorganic compounds. Chichester, Butterworth, 1975. 277p.

GOMES, C.F. Argilas - O que são e para que servem. Lisboa, Fundação Calouste Gulbenkian, 1986. 457p.

INSTITUTO AGRONÔMICO DO PARANÁ - IAPAR. Disponível em <http://www.iapar.br/noticias-index/ plantio_direto.html>. Acesso em: 20 de abril de 2007.

MacCULlOCH, C. Lições do Paraná - Como o bom planejamento pode evitar o malogro urbano. Bidamérica - R. Banco Interam. Desenvol., nov/dez. de 1999. Disponível em: <http://wwww.iadb.org/idbamerica/indexportuguese.cfm>. Acesso em: 28 jan. de 2006.

MEDEIROS, C.V \& MELO, M.S. Processos erosivos no espaço urbano de Ponta Grossa. In: DITZEL, C.H.M. \& SAHR, C.L.L., orgs.. Espaço e culura: Ponta Grossa e os Campos Gerais. Ponta Grossa, Universidade Estadual e Ponta Grossa, 2001. 518p.
MERTEN, G.H. Relatório do experimento - Avaliação da erosão em sistemas de preparo e rotação de culturas em macroparcelas. Ponta Grossa, 1995.

MOORE, D.M. \& REYNOLDS JR., R.C. X-Ray diffraction and the identification and analysis of clay minerals. 2.ed.. New York, Oxford University Press, 1997. 378p.

MORI, P.; REEVES, S.; CORREIA, C.T. \& HAUKKA, M. Development of a fused glass disc XRF facility and comparison with the pressed powder pellet technique at Instituto de Geociências, Sao Paulo University. R. Bras. Geoci., 29:441-446, 1999.

PAULETTI, V.; LIMA, M.R.; BARCIK, C. \& BITTENCOURT, A. Rendimento de grãos de milho e soja em uma sucessão cultural de oito anos sob diferentes sistemas de manejo de solo e de culturas. Ci.. Rural, 33:491-495, 2003.

REATTO, A.; FONTES, M.P.F.; ALVAREZ V., V.H.; RESENDE, M.; KER, J.C. \& COSTA, L.M. Caracterização mineralógica, potencial de reserva e sustentabilidade agrícola de alguns sítios florestais de eucalipto da região do Vale do Rio Doce (MG). R. Bras. Ci. Solo, 22:255-266, 1998.

RHEINHEIMER, D.S.; KAMINSKI, J.; LUPATINI, G.C. \& SANTOS, E.J.S. Modificações em atributos químicos de solo arenoso sob sistema plantio direto. R. Bras. Ci. Solo, 22:713-721, 1998.

RHEINHEIMER, D.S.; KAMINSKI, J.; SANTOS, E.J.S.; GATIBONI, L.C. \& BORTOLUZZI, E. Alterações de atributos do solo pela calagem superficial e incorporada a partir de pastagem natural. R. Bras. Ci. Solo, 24:797-805, 2000.

RIBON, A.A.; CENTURION, J.F.; CENTURION, M.A.P.C. \& CARVALHO FILHO, A. Propriedades físicas de Latossolo e Argissolo em função de práticas de manejo aplicadas na entrelinha da cultura da seringueira (Hevea brasiliensis). R. Bras. Ci. Solo, 26:781-787, 2002.

SCHAEFER, C.E.G.R.; SOUZA, C.M.; VALLEJOS, M.F.J.; VIANA, J.H.M.; GALVÃO, J.C.C. \& RIBEIRO, L.M. Características da porosidade de um Argissolo VermelhoAmarelo submetido a diferentes sistemas de preparo de solo. R. Bras. Ci. Solo, 25:765-769, 2001.

SILVA, C.C. \& SILVEIRA, P.M. Influência de sistemas agrícolas em características químico-físicas do solo. Ci.. Agrotec., 26:505-515, 2002.

SILVA, I.F. \& MIELNICZUK, J. Sistemas de cultivo e características do solo afetando a estabilidade de agregados. R. Bras. Ci. Solo, 22:311-317, 1998.

SILVA, V.A.; ANDRADE, M.J.B. \& RAMALHO, M.A.P. Efeitos de métodos de preparo do solo e doses de adubação NPK sobre o feijão da "seca" em seqüência à cultura do milho. Ci.. Agrotec., 25:454-461, 2001.

WEIDLER, P.G.; LUSTER, J.; SCHNEIDER, J.; STICHER, H. \& GEHRING, A.U. The Rietveld Method apllied to the quantitative mineralogical and chemical analysis of a Ferralitic soil. Eur. J. Soil Sci., 49:95-105, 1998. 